\title{
BEAMLINE RF LOAD DEVELOPMENT AT CORNELL
}

\author{
E. Chojnacki ${ }^{\dagger}$, Cornell University, Ithaca, NY \\ W. J. Alton, Ferrite Components Inc., Hudson, NH
}

\begin{abstract}
Details of design and fabrication of a beamline RF load suited to accommodate SRF cavities are presented. Bonding an RF absorber to a heat sink is the key aspect of the load. Production loads have been effectively tested to $10.8 \mathrm{~kW}$ and operated in situ up to $3 \mathrm{~kW}$.
\end{abstract}

\section{INTRODUCTION}

Many new and proposed accelerator facilities are forging frontiers in beam intensity. The degree of intensity is relative to each type of accelerator (circular, linear, $\mathrm{CW}$, pulsed) and the applications range from high energy physics with diminishing event cross sections to brighter and shorter wavelength light sources to nuclear transmutation drivers. One consequence of high beam intensity is increased excitation of destructive higher-order RF modes (HOM's) in the accelerating structure. RF feedback can damp these instabilities to a certain extent, but aggressive resistive damping is required to avoid inordinate feedback power levels.

The superconducting RF (SRF) program at Cornell has long anticipated the need for ever-increasing beam intensity [1]. The large aperture, low-impedance, and high $Q$ of SRF cavities are ideally suited to intense beams. Modest tailoring of the beampipe near an SRF cavity iris allows all HOM modes to leak out of the cavity and propagate down the beampipe. It was thus natural to place HOM RF loads directly on the beampipe in close proximity to the SRF cavity [2]. The current Cornell HOM load design has been effectively tested up to $10.8 \mathrm{~kW}$ per load, with two loads typically associated with each cavity [3]. SRF cavities requiring less aggressive HOM damping utilize a coaxial antenna coupled to the beampipe.

Fabrication of beamline HOM loads can be challenging, the most difficult task being bonding RF absorbing material to a heat sink. For this reason, the Cornell design evolved to modular panels containing the RF absorbing element. The panels are individually prepared, tested, then installed into a beampipe as shown in Fig. 1. Described below are materials, processes, and tests required to fabricate an HOM load developed over several years at Cornell with numerous instances of outside consultation.

\section{RF ABSORBER}

The close proximity of the load to the accelerating cavity places numerous demands on the load, particularly the RF absorber, as stipulated in Ref. [2]. Among commonly known RF absorbing materials, two that satisfy beamline requirements are unbiased ferrites, such as nickel-zinc, and

\footnotetext{
* Work supported by NSF and DOE STTR Grant No. DE-FG0296ER86049.

† Email: epc1@cornell.edu
}

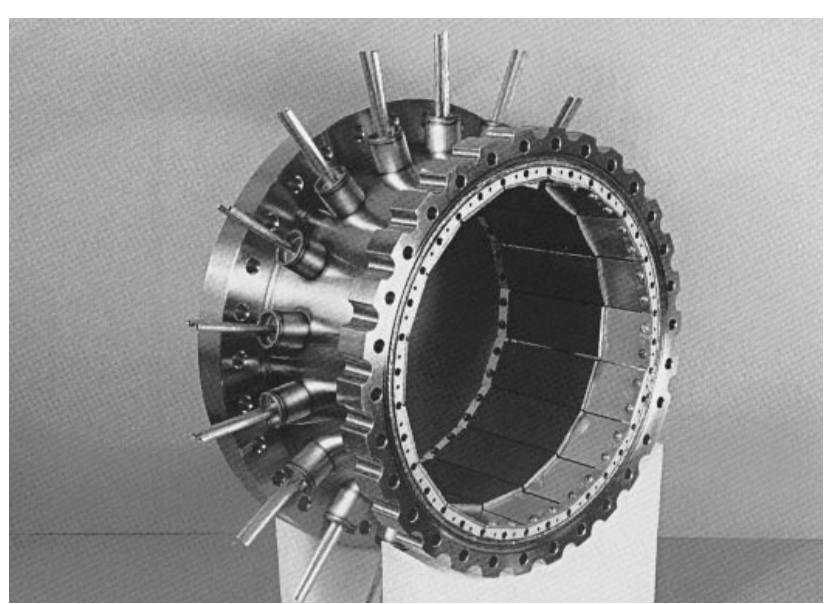

Figure 1. A Cornell HOM load.

lossy dielectrics, such as silicon carbide. Ferrites have the advantage of complex permeability $\mu$ as well as complex permittivity $\varepsilon$. Complex $\mu$ is necessary since the absorber located on the wall must have strong interaction with the magnetic field characteristic of most HOM's.

A ferrite satisfying beamline requirements and having an absorption frequency range covering the CESR III SRF cavity HOM spectrum is identified in Ref. [4], although other manufacturers of similarly acceptable ferrites exist. Complex $\mu$ and $\varepsilon$ of such materials have been determined by thorough measurement [5].

\section{BONDING AND HEAT SINK}

Heat generated by RF in the ferrite must have a highconductance thermal path to a sink. Thus the ferrite must have a high-conductance bond to a cooled, good thermal conductor.

\subsection{Soldering}

Bonds based on adhesives satisfy neither ultra-high vacuum nor thermal conductivity requirements, and simple mechanical clamping has insufficient thermal conductivity. One of the best bonds from a thermal conductance point of view is to apply a metal plating to the ferrite and solder it to a water-cooled metal substrate. Unfortunately, therein lies a problem fundamental to ceramic bonding: the ferrite's coefficient of thermal expansion is about half that of copper or other solderable metals, causing the ferrite to readily fracture during the soldering thermal cycle or when heated by RF in service. Further, soldering must be performed fluxless to preserve the porous ferrite's ultra-high vacuum integrity.

Successful solder bonds can indeed be accomplished between plated ferrite and copper if the copper is thoroughly annealed $\left(>800^{\circ} \mathrm{C}\right.$ in a vacuum furnace) prior 
to soldering, conferring it a very low mechanical yield point. However, the "dead-soft" copper must not be work hardened, in even the slightest fashion, forever thereafter. Otherwise, the induced mechanical stress in the copper will fracture the ferrite the first time it is heated.

\subsection{Elkonite}

Obviously, a heat sink material with thermal expansion coefficient similar to ferrite avoids these thermal expansion problems. The material must also be solderable and preferably have a low mechanical yield point to forgive slight differences in thermal expansion or work hardening. The only candidate found to date is a material called Elkonite [6]. Elkonite is made by sintering tungsten to a specified porosity, then filling the refractory metal with molten copper to form a nearly fully dense, vacuum-tight body. The final coefficient of thermal expansion is approximately the volume average of the composites, the available ratio $58 \% \mathrm{~W}$ and $42 \% \mathrm{Cu}$ nearly matching ferrite's nominal $9 \times 10^{-6} /{ }^{\circ} \mathrm{C}$. Beside thermal expansion matching, Elkonite is rugged and much more tolerant than $\mathrm{Cu}$ to rough handling. In its delivered form, Elkonite does not wet as well to solder as pure $\mathrm{Cu}$, thus it is electroplated with 0.001 " of $\mathrm{Cu}$ onto its surface. This layer of $\mathrm{Cu}$ being very thin and fully annealed (from the braze step described in Section 3.4) does not negate Elkonite's desirable properties. The electroplater should be aware that Elkonite is sintered and the plating operation must not allow it to disintegrate in the acid solution [7].

\subsection{Ferrite Tile Geometry}

Once bonded, a ferrite tile heated by RF is mechanically stressed so as to convex the exposed face, also known as a "crowning". The peak mechanical stress familiarly occurs near corners of rectangular tiles, and ferrite fractures experienced with $\mathrm{Cu}$ heat sinks predominantly originated in corners. Peak stress is minimal in a circular ferrite tile and is reduced by rounding the corners of a rectangular tile. The smaller the diameter of a circular tile, the lower the accumulated thermo-mechanical stress, and the higher the allowable absorbed RF power density. A lower limit in tile diameter is set by having to prepare and solder more tiles to obtain a desired surface area, especially given the typical $75 \%$ yield of successful solder bonds as discussed in Sections 4.1 and 5.

\subsection{Coolant Interface}

The next link in the heat-sink circuit is coolant. For the modular load shown in Fig. 1, cooling water flows in a copper tube brazed to the face of the Elkonite opposite the ferrites. Naturally, the difference in thermal expansion between $\mathrm{Cu}$ and Elkonite warps the Elkonite during the high temperature braze as well as the low temperature ferrite solder. Thus, the brazing and soldering fixtures must incorporate rigid clamping of the Elkonite to minimize warping. The cooling tube braze is performed in a vacuum furnace, using Cusil as filler with a $780^{\circ} \mathrm{C}$ eutectic liquidus, typically heated to $820^{\circ} \mathrm{C}$ with a 10 minute soak at temperature. After the braze, the assembly is tamped onto a flat surface to restore Elkonite flatness prior to ferrite soldering. The sintered $\mathrm{W}$ and annealed $\mathrm{Cu}$ easily yield, yet do not causing work-hardening problems encountered with pure $\mathrm{Cu}$.

\section{FERRITE PROCESSING}

Ferrite tiles are typically cleaned with alkaline solution by the manufacturer, and upon delivery ultrasonically cleaned in methanol baths, 6 cycles of 15 minutes each with changing of the methanol between cycles. Tests showed that after the 3rd ultrasound bath, no more particulate leaves the ferrite of Ref. [4]. The ferrites are then fired in air with $100^{\circ} \mathrm{C} / \mathrm{hr}$ ramp up to $900^{\circ} \mathrm{C}, 2 \mathrm{hr}$ soak at $900^{\circ} \mathrm{C}$, and $100^{\circ} \mathrm{C} / \mathrm{hr}$ ramp down. Air firing reduces later vacuum outgassing and is reputed to help homogenize the ferrite.

\subsection{Plating}

The ferrites are next plated with a solderable material. Since nickel is a primary constituent of ferrite, it was reasoned that sputtered $\mathrm{Ni}$ would have good adhesion. A problem with this plating has been spots of complete "scavenging" off the ferrite by the molten solder. In Cornell HOM load production, about $25 \%$ of ferrite solders exhibit scavenging problems. The thicker the plating the less scavenging is observed, but sputtering is a slow process and too thick a plating builds surface tension that may shear the ferrite. Nickel-vanadium can be sputtered at a higher rate than pure $\mathrm{Ni}$, thus to date Cornell production has targeted $25000 \AA$ of Ni-V.

A promising alternate plating is a layered approach whereby $300 \AA$ of titanium is first sputtered, then a $2000 \AA$ graded layer of $\mathrm{Ti}$ and $\mathrm{Cu}$ (requiring two sputter guns), then $5000 \AA$ of pure $\mathrm{Cu}$, ending with a gold flash to inhibit oxidation [8]. The reasoning behind this plating is that $\mathrm{Ti}$ adheres very strongly to the ferrite, the Ti-Cu composite retains $\mathrm{Cu}$ from scavenging, then the $\mathrm{Cu}$ wets well to solder though some may be scavenged. There have not been statistically significant tests of the $\mathrm{Ti}-\mathrm{Cu}$ graded plating to date, but a few samples have soldered well.

\subsection{Soldering}

From thermal stress considerations, ferrite soldering is performed at as low a temperature as possible while providing a safety margin from solder melting during HOM load operation. The majority of Cornell soldering has used $90 \% \mathrm{Sn} / 10 \% \mathrm{Ag}$ filler having a $221^{\circ} \mathrm{C}$ solidus and $295^{\circ} \mathrm{C}$ liquidus. The 0.004" $\mathrm{Sn}-\mathrm{Ag}$ foils are sandwiched between the ferrite and Elkonite, then heated to $370^{\circ} \mathrm{C}$ in a vacuum furnace with several intermediate soaks and a 10 minute soak at temperature. Generous spacing among fixtures prevents shadowing of radiative heat transfer, providing uniform fixture temperatures. There is no gas backfilling of the furnace, the slow cool (>8 hrs) allowing the solder to noticeably crystallize, with loose flakes later removed by wire brushing. Tests with argon and nitrogen gas backfilling after the heat cycle have yielded very poor bonds, with nearly all the Ni-V plating being scavenged and the ferrite readily falling off. Perhaps the gas backfill enables such a good bond to Ni-V that the plating is completely cleaved off the ferrite, and vacuum cooling with crystallization simply yields numerous 


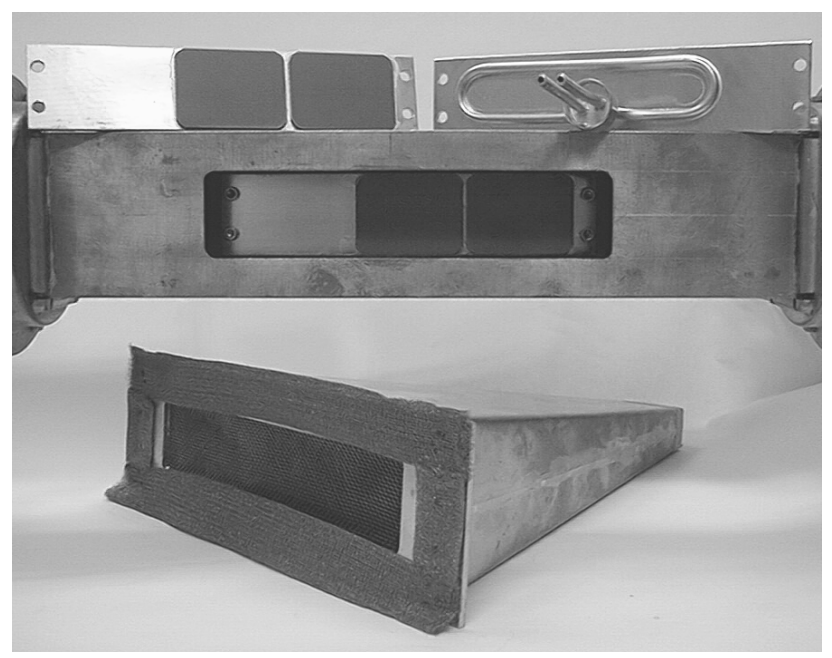

Figure 2. HOM load individual panel test setup.

islands of adhesion. Details of such chemistry and mechanics remain to be studied.

An alternate solder filler is $80 \% \mathrm{Au} / 20 \% \mathrm{Sn}$ with a $280^{\circ} \mathrm{C}$ eutectic liquidus. Au-Sn wets better than Sn-Ag, although the foil is less malleable at the outset. There have not been statistically significant tests of the Au-Sn filler to date, and among a few samples one case had a poor bond. Thus, the added expense of Au remains to be justified.

\section{RF TESTS}

Individual panels of Elkonite with bonded ferrite are tested in an RF setup pictured in Fig. 2. The panels are mounted on a broad wall of WR-284 waveguide, fed by up to $1 \mathrm{kWCW}$ at $2450 \mathrm{MHz}$ by a magnetron, with a sliding short downstream to adjust the standing wave along the ferrite. Water flows through the cooling tube at $0.6 \mathrm{GPM}$. The panels are viewed with an infra-red camera through a hole in the opposite wall of the waveguide, covered by a screen and RF evanescent cone, also shown in Fig. 2. Areas of poor solder bond are evident by heating in excess of the standing wave pattern as seen by the IR camera, an example of which is shown in Fig. 3. Thermal transients at turn-on and turn-off of RF are also excellent diagnostics, areas of even slightly poor solder bond showing thermal lag.

The RF power in the test is typically limited to $600 \mathrm{~W}$, corresponding to an average power absorption density of $15.5 \mathrm{~W} / \mathrm{cm}^{2}$ on each of two $5.08 \mathrm{~cm} \times$ $3.81 \mathrm{~cm} \times 0.32 \mathrm{~cm}$ ferrite tiles. The RF standing wave pattern scanned over the ferrite raises regions up to twice this power density, but such inhomogeneous heating probably also occurs in service [9]. With 18 such assemblies per load, the assembled unit is effectively tested up to $10.8 \mathrm{~kW}$ average HOM power, and two loads are connected to each SRF cavity [3]. The upper limit in absorbed power density for a few test assemblies has been $25 \mathrm{~W} / \mathrm{cm}^{2}$, at which point the ferrite cracked. This power density could be raised by use of circular tiles with small diameter and a broader cooling tube attachment. As

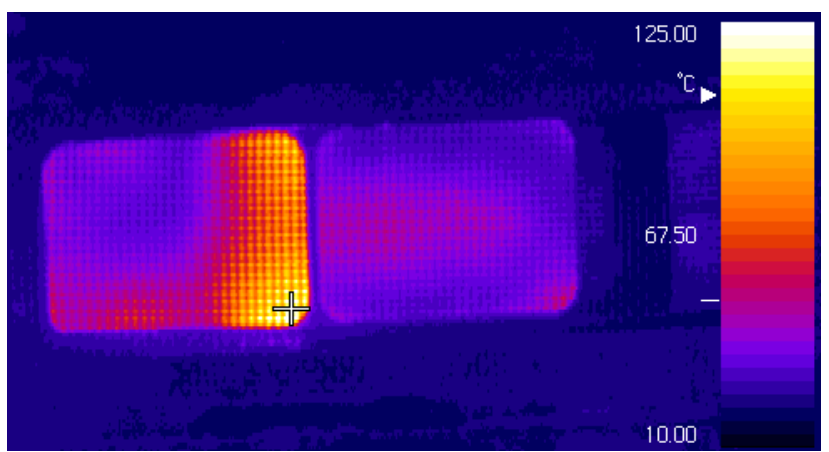

Figure 3. Infra-red image from the test setup exhibiting a hot area due to poor solder bond. The grid pattern is due to the screen covering the waveguide hole.

mentioned in Section 4.1, $25 \%$ of the panels are rejected due to "hot spots", later diagnosed as scavenged plating.

\section{FINAL ASSEMBLY}

Elkonite/ferrite panels that pass the RF test are inserted into a stainless steel beamline housing as shown in Fig. 1. The panels are tightly fastened with screws to provide good electrical grounding to accommodate beam image wall currents. Direct interaction of beam fields with the ferrite has been demonstrated to be negligible [10]. Brazed fittings that incorporate the cooling tubes for each assembly are then welded into the steel housing to provide a vacuum-tight enclosure. The finished unit is rinsed with methanol, the screws temporarily loosened, and the unit vacuum baked at $150^{\circ} \mathrm{C}$ for several days. After baking, the room temperature vacuum bottoms out at $<10^{-9} \mathrm{~T}$. Upon HOM load attachment to the SRF cavity beamline, the panel screws are re-tightened. Performance of six such prepared loads has been quite satisfactory, dissipating up to $3 \mathrm{~kW}$ per load to date [3].

\section{REFERENCES}

[1] M. Tigner, "Imperatives for Future High Energy Accelerators", AIP Conf Proc 279, Advanced Accelerator Concepts (Port Jefferson, NY, 1992), p. 1.

[2] D. Moffat, et. al., "Design and Fabrication of a Ferrite-lined HOM Load for CESR-B", Proc. 1993 Part. Accel. Conf. (Washington, DC, 1993), p. 977.

[3] S. Belomestnykh, et. al., "Commissioning of the Superconducting RF Cavities for the CESR Luminosity Upgrade", these proceedings.

[4] C-48, Countis Industries, Carson City, NV, (775) 882-6123.

[5] W. Hartung, et. al., "Measurements of the Microwave Properties of Some Absorbing Materials", Proc. Workshop on MicrowaveAbsorbing Materials for Accelerators (Newport News, VA, 1993), p. 162.

[6] 10W3 Elkonite, CMW Inc., Indianapolis, IN, (317) 634-8884.

[7] Anoplate Corp., Syracuse, NY, (315) 471-6143.

[8] JP Argana Contract Services, Caledonia, NY, (716) 538-4682.

[9] B. Vakoc, "HOM Spectra and Dissipated Power Density Profiles for the SRF Cavity with a Ferrite HOM Load", Cornell LNS Report SRF 950811-11 (1995).

[10] W. Hartung, et. al., "Measurement of the Interaction between a Beam and a Beam Line Higher-Order Mode Absorber in a Storage Ring", Proc. 1995 Part. Accel. Conf. (Dallas, TX, 1995), p.3294. 